\title{
Virgo and the quest for gravitational waves
}

\author{
André-Claude Clapson and Patrice Hello, \\ Laboratoire de I'Accélérateur Linéaire IN2P3/CNRS and Université de Paris XI Campus d'Orsay • 91898 Orsay Cedex • France
}

\begin{abstract}
Tn the past ten years, several giant interferometers have been built 1 around the world with the goal of a first direct detection of gravitational waves. The most sensitive detectors, 2 interferometers for the US LIGO collaboration and the detector built by the Italo-French collaboration Virgo (fig. 1) are approaching their design sensitivity. Scientific exploitation of these instruments is now starting ...
\end{abstract}

\section{What is a Gravitational Wave?}

Basically, Gravitational Waves (GW) are ripples of space-time, which propagate at the speed of light. They are predicted by general relativity and other, alternative, modern theories of gravitation, as an effect of accelerated motions of matter. This is somewhat similar to electromagnetic waves, produced by the motion of accelerated charges. A difference is that GW production requires non symmetrical distributions of matter. Thus a star collapsing with perfect spherical symmetry emits no GW. Another difference is that at the lowest order GW are quadrupolar waves while electromagnetic waves are dipolar.

An observable indication of the passage of these ripples of space-time is a modification of the measures of distance. More precisely, consider a circle of test masses at rest (Fig. 2). As a GW with normal incidence passes through the circle distances in one direction seem to expand while in a perpendicular one they seem to shrink, owing to the quadrupolar nature of GW. This relative distance modification is used in the definition of the GW dimensionless amplitude: $h(t) \approx \frac{1}{2} \frac{\delta L}{L}$ where $L$ is the distance measured in the absence of GW (the ordinary flat space-time). The problem is that $h$ is in general so weak that detecting a GW seems hopeless.

\section{Astrophysical sources}

In fact, only sources involving relativistic (compact) stars such as black holes or neutron stars may emit GW that can be detectable on Earth. The most promising sources are thought to be binary systems of inspiralling neutron stars or black holes. In such a system emitted GW carry away part of the orbital energy. This loss entails a decrease of the orbital radius: with time the two stars become closer and closer and as they get closer the GW emission becomes more and more intense. The resulting GW signal $h(t)$ is called a chirp, a sinusoid with increasing amplitude and frequency

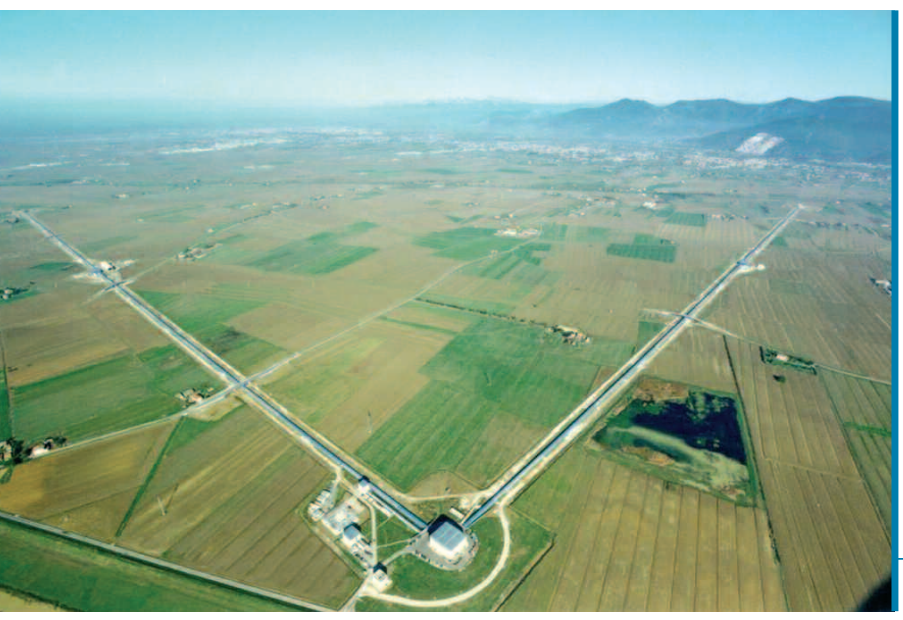

that ends when the two objects merge. Collapses of massive stars into neutron stars or black holes (gravitational supernovae) are also sources of GW that must be considered. Here typical GW signals (though very difficult to model) are bursts with estimated typical duration of the order of a few milliseconds. Finally let's mention other kinds of sources, such as isolated rotating neutron stars or pulsars that can emit weak continuous signals if the star is not perfectly symmetric or cosmological backgrounds.

A survey of potential GW sources, orders of magnitude for amplitudes and rates, leads to a simple conclusion. A ground detector of GW must be designed to be sensitive to GW amplitudes of typically $h \sim 10^{-21}$ or less, in a bandwidth from $10 \mathrm{~Hz}$ up to a few thousands $\mathrm{Hz}$ to be able to observe these waves one day. This is the goal of the first generation GW interferometers that are in (or are entering into) operation today.

\section{The interferometric detection of Gravitational Waves}

The hunt for Gravitational Waves is not a recent story. It began at the end of the 50's under the impulse of the American physicist Joe Weber. The GW detector he invented was a cylindrical bar about 1.5 meters long equipped with piezo-electric transducers to record the vibrations of the bar, to be excited by the passing GW. This setup spawned several modern descendants: aluminium bars (and more recently spheres) cooled below $1 \mathrm{~K}$. The main limitation of this kind of detector is its very narrow bandwidth (typically a few $\mathrm{Hz}$ ). Detectors based on optical interferometry are not limited in this way.

Interferometric detection relies on the Michelson interferometer scheme (see fig. 3). The output port power is given by $P_{\text {mich }}=\frac{P_{0}}{2}\left[1+C \cos \left(2 \frac{\omega_{0}}{c} \Delta l_{\text {arms }}\right)\right]$ with $P_{0}$ the input laser power, $\omega_{0}$ the laser angular frequency, $\Delta l_{\text {arms }}$ the optical path difference between the arms and $C$ the interference contrast, as set by the parameters of the optical cavity. The interferometer must be tuned in order to optimize the sensitivity to a gravitational wave, that is to say to small variations in $\Delta l_{\text {arms. }}$. The main fundamental noise limiting the sensitivity is shot noise. It imposes the dark fringe, where $P_{\text {mich }}$ is minimized, as the best configuration. In order to improve sensitivity, we can lower the shot noise. Only two parameters are effective: the optical length of the interferometer arms and the power circulating in the interferometer. This is why interferometric detectors have kilometric arms ( $3 \mathrm{~km}$ in the case of Virgo). An optical path of about 100 $\mathrm{km}$ can then be obtained by placing a Fabry-Perot cavity in the arms. Concerning the light power, as continuous laser sources provide limited power, the trick consists in the addition of an extra mirror at the input of the interferometer. This mirror and the rest of the interferometer create a new cavity (power-recycling cavity) where the stored light power is enhanced at resonance (See fig. 3 for the final optical design of a Virgo-like detector).

A shot-noise limited spectral sensitivity of the order of $10^{-23} \mathrm{~Hz}^{-1 / 2}$ can be achieved using the complete setup with Virgo dimensions: 3 $\mathrm{km}$ Fabry-Perot arm cavities with a finesse of 50, a $10 \mathrm{~W}$ input laser beam and a recycling power gain of the order of 50 . Of course other noises are likely to limit the sensitivity in diverse spectral zones. 
Fundamental, environmental and technological noises The first obvious obstacle to low frequency measurements in a Michelson interferometer is seismic noise. Measurements of displacement spectral densities in quiet sites typically give $\tilde{x}(f) \approx 10^{-6} / f^{2}$ meters $/ \sqrt{\mathrm{Hz}}$.

The mirrors must therefore be isolated from ground motions. This can be achieved by suspending them. Basically the suspended mirrors then behave like harmonic pendulums with a natural angular frequency of oscillation $\omega_{0}=\sqrt{g / l}$, where $g$ is the gravitational acceleration and $l$ the pendulum length. While ground motion is amplified at the natural frequency, it is by contrast damped by $\omega_{0}^{2} / \omega^{2}$ at higher frequencies. In Virgo the mirrors are suspended in a multiple-stages pendulum (fig. 4) with a fundamental resonance below $1 \mathrm{~Hz}$ and a resulting seismic attenuation factor of the order of $10^{10}$ at $10 \mathrm{~Hz}$.

Thermal noise is another annoying source of noise. Indeed mirrors and their suspensions all are mechanical resonators, whose degrees of freedom are excited at ambient temperature. The alternative to cooling the whole instrument is to select high quality-factor materials, in particular for the mirror substrates and suspension wires, in order to limit the spectral spread of the noise. Besides, sources of mechanical losses must be hunted.

Laser noises must be also controlled. In particular fluctuations in laser frequency or power can couple to interferometer length asymmetries to give dangerous phase noises than can compete with the shot noise. The laser is therefore actively servoed on a length reference, provided by an Ultra Low Expansion (ULE) cavity, and therefore frequency-stabilised. Before entering the interferometer itself, the laser beam passes through another Fabry-Perot cavity, named the Mode-Cleaner. A cavity is indeed an optical resonator so it filters off-resonance beam fluctuations (more precisely it is a low-pass filter). This helps to attenuate the laser fluctuations, including beam jitters.

Ultra high vacuum is of course required in order to avoid spurious signals due to residual gas index fluctuations in the interferometer arms. The vacuum level specification corresponds to a residual pressure below $10^{-7}$ mbar. The interferometer must be entirely under vacuum. Hence the mirrors and their suspensions are located in towers and the kilometric arms are in the material form of tubes (120 cm diameter in Virgo).

The inner parts of the tubes and towers are equipped with baffles, whose function is to remove the light scattered by mirrors in offaxis directions. Mirrors always have some residual rugosity, in spite of their very good quality. This rugosity causes a small part of the beam photons to travel off-axis and eventually reflect on the infrastructure (tubes, towers) which is not isolated from seismic noise. These photons then get random phases and the recombination of some of them with the main beam produces an extra phase noise which would be at the level of the shot noise if nothing were done!

The design sensitivity curve of Virgo is shown in figure 5. All technological noises are supposedly under control. The sensitivity is then limited at low frequency by the seismic wall (seismic noise below $4 \mathrm{~Hz}$, not filtered by the super attenuator), at intermediary frequencies by the thermal noise (the high frequency tail of the pendulum thermal noise, the low-frequency tail of the mirrors' thermal noise plus the many resonances due to the suspension wires) and in the high frequency region by the shot noise.

\section{Where are we?}

The construction of Virgo was achieved in fall 2003. Since then the detector has been in the commissioning phase. Virgo is a very complex instrument and reaching the design sensitivity takes time. One of the main tasks is the control of the interferometer itself.
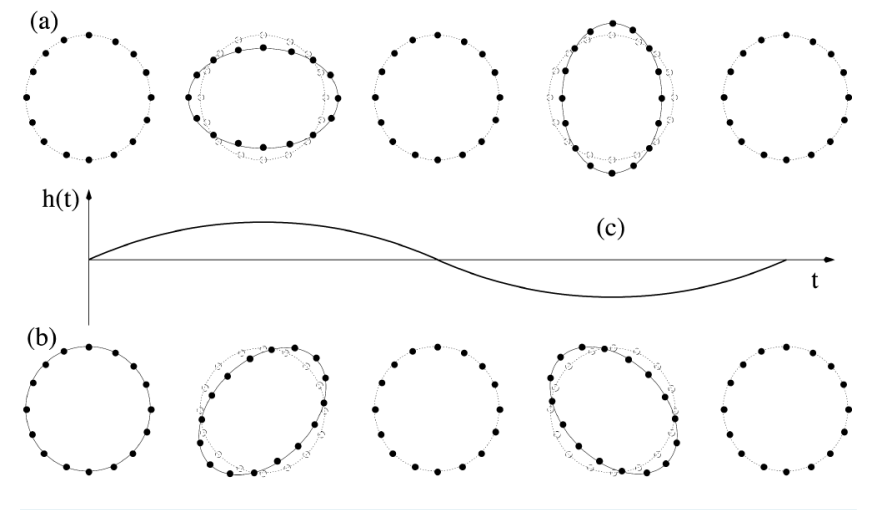

$\Delta$ Fig. 2: Effect of a GW passing through a circle of test masses. The wave incidence is normal to the mass plane. (a) the effect of a "+" polarised wave. (b) the effect of a " $x$ " polarised wave. The amplitude of the GW $h(t)$ is shown as a function of time $t$.

When free, suspended mirrors oscillate continuously at the pendulum resonance frequency. The working point of the detector is obtained when arm cavities are locked at resonance for the laser beam, the Michelson interferometer tuned at a dark fringe and the recycling cavity also resonant for the laser beam. This gives four optical lengths to be controlled, plus the mirror rotation degrees of freedom. We see that Virgo needs an active servo-system to control lengths and angular tilts of mirrors as far as $3 \mathrm{~km}$ apart, on a time scale of a fraction of second.

The commissioning has been carried out step by step, locking first the one-arm cavities, then the two arms plus the Michelson interferometer and finally the complete configuration. The automatic alignment (angular control) has been built and tested in parallel.

To date the Virgo sensitivity is roughly one order of magnitude above the design curve. Meanwhile the two $4 \mathrm{~km}$ detectors of the US LIGO collaboration have reached more or less their design sensitivity above $100 \mathrm{~Hz}$ (commissioning started earlier for LIGO).

Since the data produced by these instruments are mostly composed of noise, with hopefully someday an astrophysical signal, the first challenge for analysis is to distinguish signal from noise. Otherwise, we would be swamped by false GW detections, to be identified as such by eye. Most of the efforts so far have focussed on inspiral and burst scenarios, though all avenues are explored. Many types of algorithms have been tested, some looking for specific waveforms that general relativity may provide, others aiming at generic signal signatures. As short duration signals are easily mimicked by the instrument, successful analysis is strongly coupled to an understanding of the behaviour of the instrument.

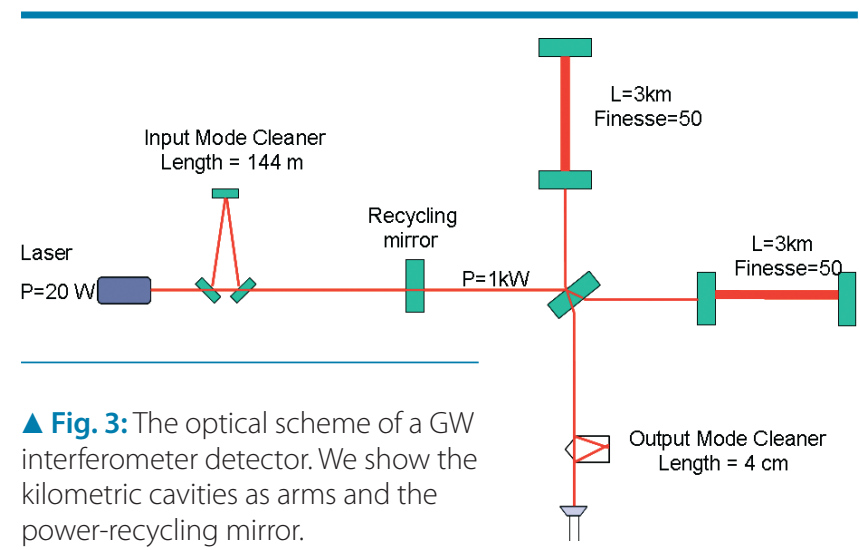




\section{The future}

The question is not if we will detect GW but rather when! Evidence for their existence is provided by the study of the binary pulsar PSR B1913+16 discovered by the Nobel laureates Hulse and Taylor in the seventies. This inspiralling system consists of two neutron stars, one being a pulsar detected by radio-telescopes. This is a perfect example of a GW emitter. Astrophysicists have been able to compare the behaviour of the binary pulsar to the predictions of general relativity. Concerning the orbital decay due to GW emission, measurements and theory agree within less than $1 \%$. This is a beautiful proof that GW exist!

Of course physicists are now waiting for a direct detection. For the very near future it is likely that three (two for LIGO and one for Virgo) detectors with similar sensitivities will be on watch for a wave to come. With some luck, smaller instruments (the AngloGerman GEO 600 or the Japanese TAMA 300) may also contribute to the discovery.

To increase the chance of detection and to confirm detection, a network of detectors is necessary. Validation of detection can be achieved by studying coincident events with other types of

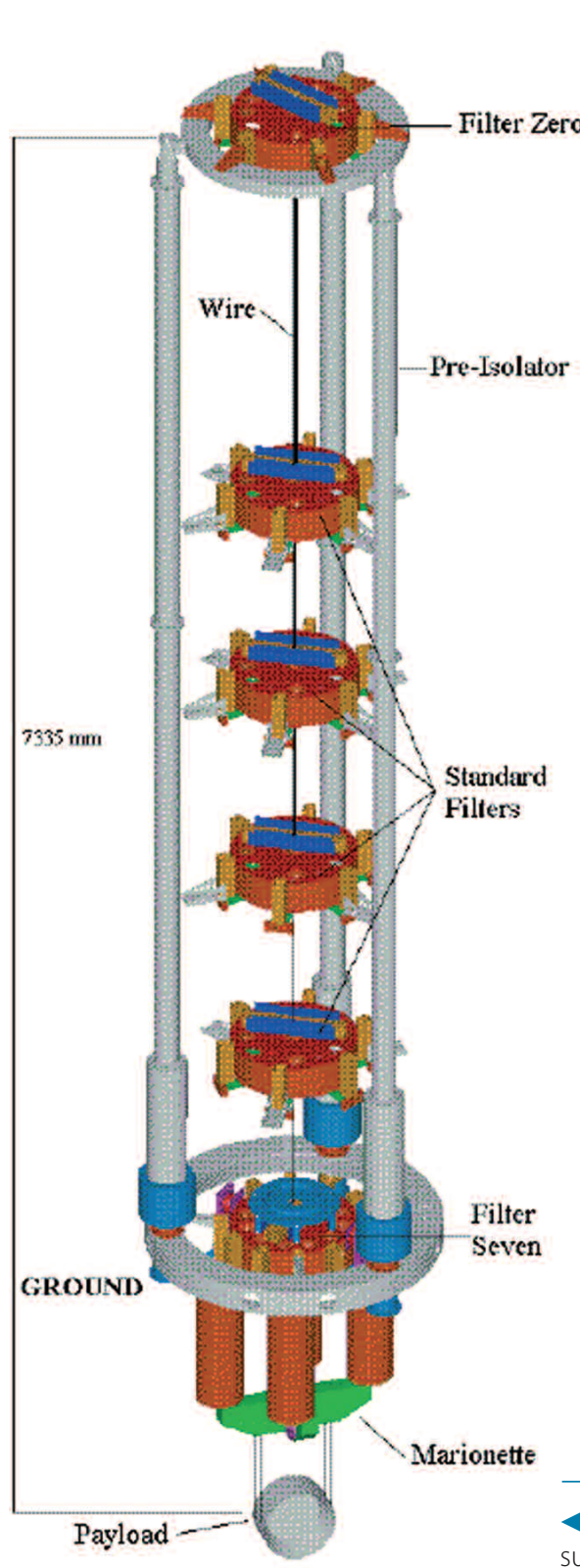

detectors, such as neutrino detectors or Gamma-Ray satellites, since these signals are likely to be the counterparts of GW emitters, neutrinos in the case of a supernova event or Gamma Ray bursts in the case of the merger of neutron stars. However, to obtain a complete reconstruction of the passing waveform (amplitude, polarisation, location of
Fig. 4: The Virgo super attenuator. the source ...) at least three GW detectors are mandatory. Virgotype instruments are indeed not directional as traditional telescopes. Their antenna pattern looks somewhat like a peanut, symmetrical with respect to the interferometer plane. With coincident events from three GW detectors at separate locations, source location can be reconstructed using triangulation. More details could be obtained through sophisticated coherent analysis methods.

Nevertheless even with three detectors (LIGO and Virgo) working at their design sensitivity a first direct detection in the near future is not guaranteed. Due to the uncertainties in rates of events (binary inspirals) or in waveform amplitudes (supernovae), it is believed today that to be sure to "see" several events per year the sensitivity of current interferometers has to be improved by about one order of magnitude. Work has already started on second generation detectors. An advanced LIGO has been funded by the NSF. R\&D for the next generation is also going on in Europe and plans for future detectors are being discussed. Of course second-generation detectors are for the next decade. Today, the GW observation field is very active. We are really at the birth of GW astronomy and a new window is about to open on the Universe.

\section{About the authors}

André-Claude Clapson is currently finishing his $\mathrm{PhD}$ thesis and Patrice Hello is Maître de Conférences at the Orsay Campus near Paris. They are both hosted by the Laboratoire de l'Accélérateur Linéaire and members of the Italian-French Virgo collaboration. They are working together mainly on Gravitational Wave Data Analysis issues. Patrice Hello has also an interest in R\&D for future Advanced Detectors of Gravitational Waves.

\section{Usefull links :}

Virgo collaboration:

www.cascina.virgo.infn.it

LIGO collaboration: www.ligo.caltech.edu

GEO600 collaboration: www.geo600.uni-hannover.de

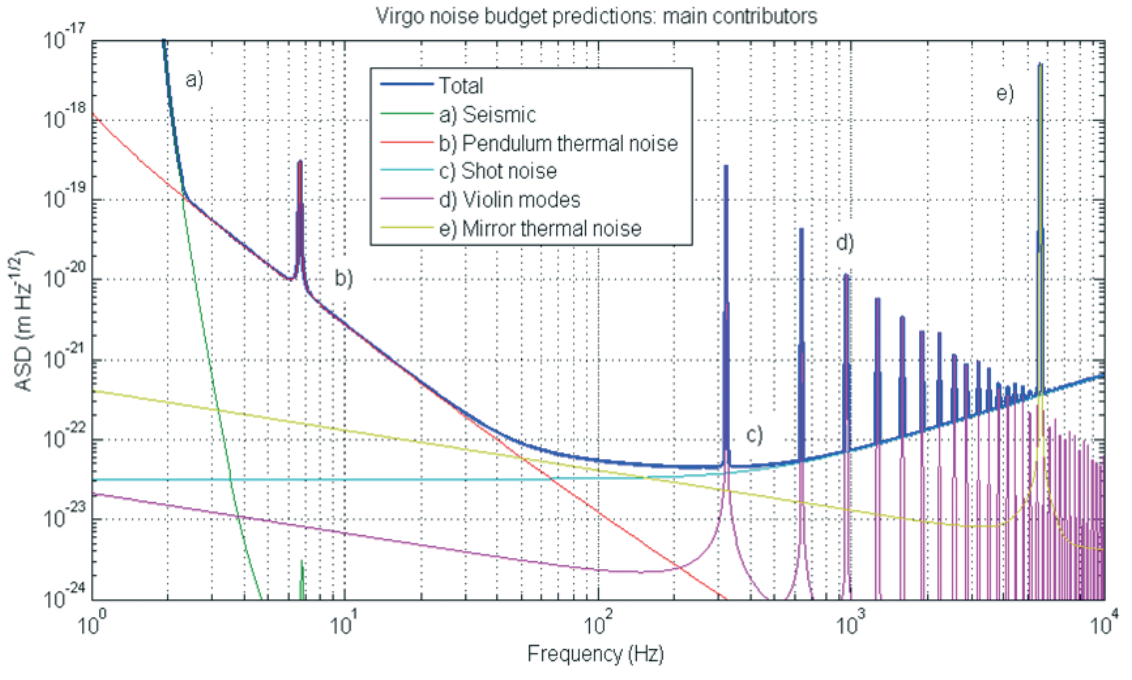

$\Delta$ Fig. 5: The Virgo design sensitivity. The sensitivity is limited in the effective bandwidth by thermal noise and shot noise. 